\title{
Spontaneous rupture of adult Wilms' tumor: A case report and review of the literature
}

\author{
Jian-lin Huang, MD; Yong Liao, MD; Yu An, MD; Ming-xing Qiu, MD \\ Department of Urology, Sichuan Academy of Medical Sciences \& Sichuan Provincial People's Hospital, Sichuan, People's Republic of China
}

Cite as: Can Urol Assoc J 2015;9(7-8):E531-4. http://dx.doi.org/10.5489/cuaj.2539

Published online July 17, 2015.

\section{Abstract}

Wilms' tumour is rare in adults, and spontaneous rupture with retroperitoneal hemorrhage as the presenting sign of renal tumour is also uncommon. We present a case of a 20-year-old woman with spontaneous rupture of Wilms' tumour by describing the course of diagnosis and treatment. The patient underwent an open left radical nephrectomy, and was treated with 18 weeks of adjuvant chemotherapy with vincristine and actinomycin D. The follow-up of 12 months demonstrated no recurrence. We also reviewed the limited number of related reports. These suggest that the preoperative diagnosis of adult Wilms' tumour is very difficult, and radical nephrectomy and postoperative comprehensive therapy are equally important in the treatment of these patients. Factors of prognosis for adults with Wilms' tumour include tumour stage, histopathology, and time and type of therapy.

\section{Introduction}

Wilms' tumour is the most common primary renal tumour in childhood, and its annual incidence rate in children under 15 years is 7 to 10 cases per million, accounting for $6 \%$ to $7 \%$ of all pediatric malignant tumours. ${ }^{1}$ It is very rare in adults, with an incidence of 0.2 per million per year in the United States and Europe. ${ }^{2}$ Spontaneous rupture of renal tumour is an uncommon event and the most frequent cause is angiomyolipoma (AML), while spontaneous rupture and hemorrhage as presenting signs of Wilms' tumour also is very rare. ${ }^{3}$ We present a case of a spontaneous rupture of adult Wilms' tumour in a 20-year-old woman with flank and back pain.

\section{Case report}

A 20-year-old woman presented to hospital with left flank pain and back pain in September 2013. She denied fever, gross hematuria, urinary frequency, urinary urgency, and urodynia. Her medical history was unremarkable and she denied any history of urinary calculus. She had no family history of urologic disorders or malignancies. Physical examination was unremarkable, except she had tenderness and percussion pain over the left kidney area. Laboratory investigations revealed no abnormalities. Renal function tests were normal and urine analysis revealed no microscopic hematuria. Renal ultrasound demonstrated a mixed-echo renal mass which was $6.4 \times 6.2 \mathrm{~cm}$ in size and the normal outline was missing at the lower pole of the left kidney. The computed tomography (CT) demonstrated a $6 \times 6-\mathrm{cm}$ heterogeneous-density mass and subcapsular hematoma in the left kidney (Fig. 1). Chest imaging and bone scanning results were both negative.

Rupture of AML with hemorrhage was considered and conservative therapy was adopted, while re-examination of CT 1 week later revealed no change, and the patient was allowed to leave the hospital. However, 2 months later the patient complained of gross hematuria, and few blood clots were contained in urine. Abdominal ultrasound revealed that the mixed-echo renal mass was $10 \times 10 \mathrm{~cm}$. A CT demonstrated a $9.8 \times 8.3-\mathrm{cm}$ mass with heterogeneous enhancement and arc-shaped subcapsular hematoma with high density (Fig. 2).

The patient was then referred to our hospital for further evaluation and treatment. On admission, her temperature was $37.1^{\circ} \mathrm{C}$, blood pressure was $124 / 84 \mathrm{mmHg}$, heart rate was $72 \mathrm{bpm}$, height and weight were $162 \mathrm{~cm}$ and $50 \mathrm{~kg}$, respectively. Physical examination revealed that the left kidney area was filled, with percussion pain. Based on the clinical findings and investigations, a preliminary diagnosis of renal carcinoma of the left kidney was made. The patient underwent an open left radical nephrectomy, with the left adrenal gland reserved through a waist incision and the operation was performed successfully.

On gross inspection, the tumour at the lower pole of left kidney measured $8 \times 6 \mathrm{~cm}$ and the hematoma was subjacent to the renal capsule. Sectioning of the tumour showed the mass had a solid and multicystic appearance with several small areas of focal hemorrhage, possible necrosis. The 


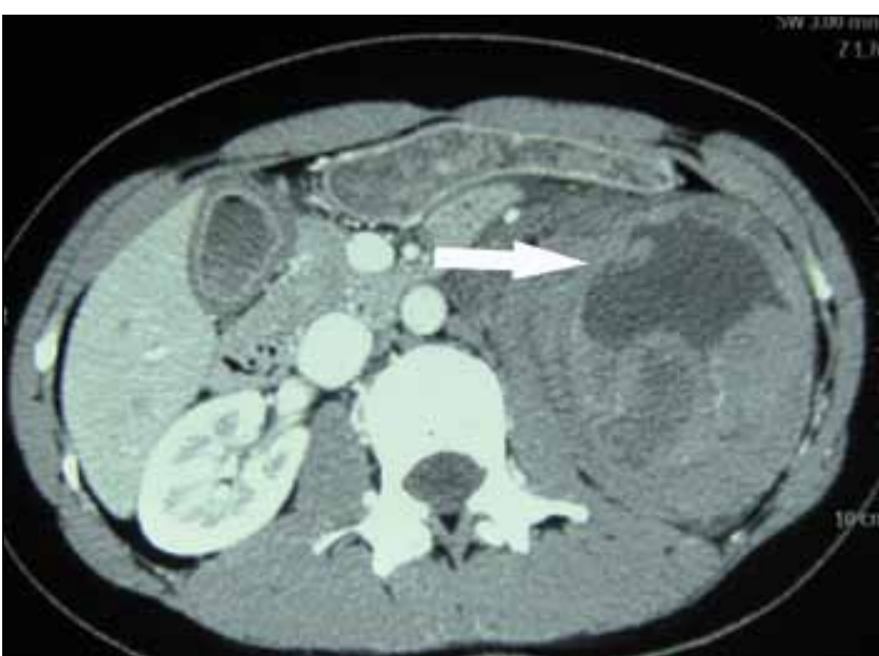

Fig. 1a. A computed tomography axial image showing a $6 \times 6-\mathrm{cm}$ heterogeneous-density mass and sub-capsular hematoma involving the left kidney.

tumour did not infiltrate the renal pelvis. The hilar lymph nodes showed no evidence of tumour. Microscopic analysis revealed that the tumour was a nephroblastoma without anaplasia (Fig. 3a, Fig. 3b). The tumour exhibited a triphasic pattern of blastema, epithelium, and stroma with myxoid background in the solid area and in the septa of the cystic area. The blastema area stained positive for vimentin, CD99 and CD117, but negative for CK, RCC, CD10, CK7, EMA, CgA, Syn, CD34, WT-1, myogenin and myoD1. All lymph nodes were negative for tumour.

The local stage was considered stage I, according National Wilms Tumor Study Group protocols. Postoperatively, the patient was treated for 18 weeks of adjuvant chemotherapy with vincristine and actinomycin $\mathrm{D}$, which she tolerated with few side effects. She has been followed 12 months after surgery. Her follow-up imaging demonstrated no recurrence, and complete remission (CR) of the tumour was achieved clinically.

\section{Discussion}

Wilms' tumour is the second most common tumour in children, accounting for $6 \%$ to $7 \%$ of all childhood tumours, ${ }^{1}$ but it is rarely found in adults. The true incidence of adult Wilms' tumour is difficult to ascertain because of its rarity in adults. A review of English literature demonstrated that fewer than 300 cases have been reported, while about another 200 cases have been reported in the Chinese literature.

The preoperative diagnosis of adult Wilms' tumour is very difficult because there are no specific radiographic findings and many other adult renal tumours that should be considered in the differential diagnosis. Pathologically, Classical Wilms' tumour has triphasic histology, with components of blastemal, epithelial, and stromal structures, as

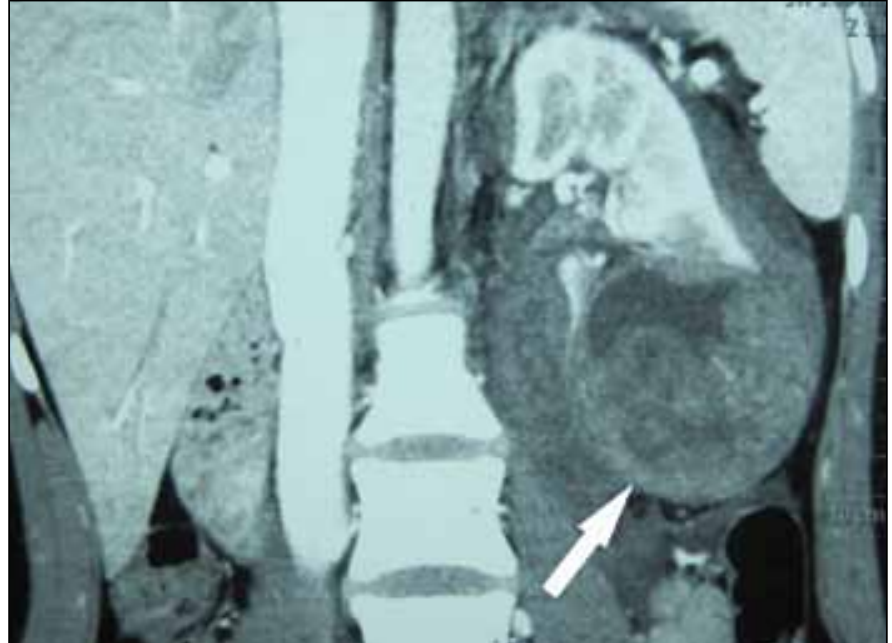

Fig. 1 b. A computed tomography coronal image showing a $6 \times 6-\mathrm{cm}$ heterogeneous-density mass and subcapsular hematoma involving the left kidney.

found in our case. In 1980, Kilton and colleagues ${ }^{4}$ developed the following criteria for the diagnosis of adult Wilms' tumour: presence of a primary renal neoplasm; presence of a primitive blastematous spindle or round-cell component; formation of abortive or embryonal, tubular epithelial or glomeruloid structures; absence of tumour diagnostic of renal cell carcinoma (RCC); pictorial confirmation of histology; and age $>15$ years. Dykes and colleagues ${ }^{5}$ reported that percutaneous needle biopsy was used in children to assess the nature of massive renal tumours; if performed before nephrectomy, percutaneous needle biopsy is $93 \%$ effective and biopsy morbidity was low. While it is difficult to differentiate between fat poor AMLs and RCC, renal mass biopsy can be considered in adults, particularly in those who are potential candidates for treatment options ranging from observation to surgical excision.

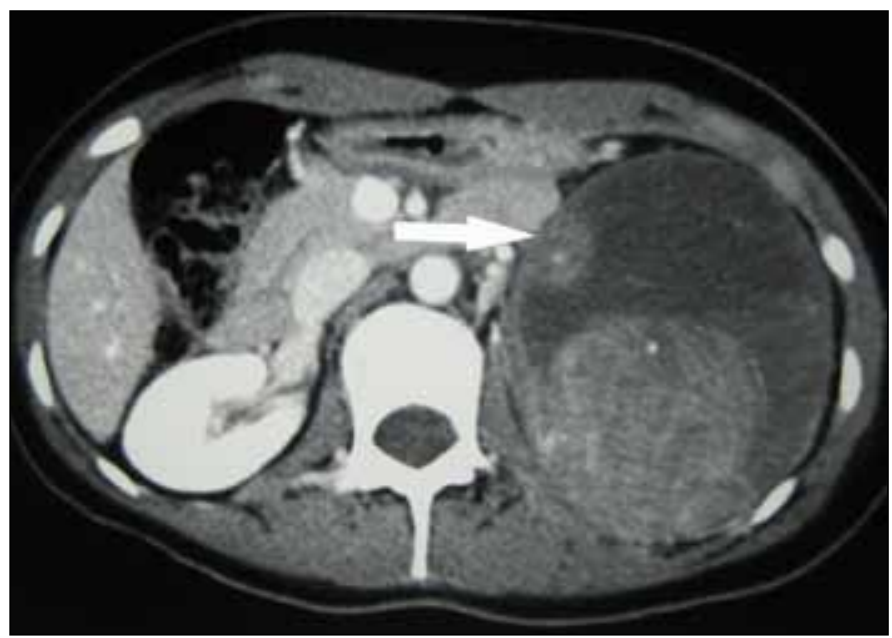

Fig. 2. A computed tomography image showing a larger mass of the left kidney $(9.8 \times 8.3 \mathrm{~cm})$ with heterogeneous enhancement 2 months later. 


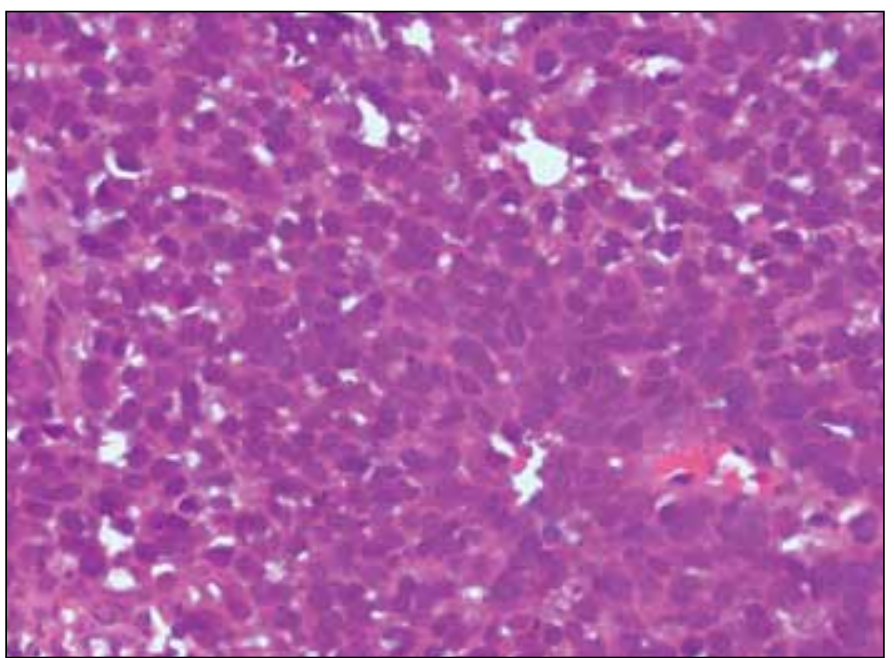

Fig. 3a. Microscopical picture (hematoxylin-eosin, $\times 400$ ) showing blastemal cells and stromal element.

Treatment guidelines in adults have not been established, most authors suggest adult patients can be treated according to pediatric protocols. ${ }^{6}$ Radical nephrectomy is the treatment of choice for one-sided nephroblastoma. Multiple studies have demonstrated that laparoscopic renal surgery provides recuperative and cosmetic advantages in contrast to open surgery. Okasho and colleagues ${ }^{7}$ reported an adult patient with Wilms' tumour who had undergone right retroperitoneoscopic nephroureterectomy because of renal pelvic carcinoma. However, some disadvantages of laparoscopic surgery exist for Wilms' tumour. The size of the tumour is usually large, and if preoperative tumour rupture happened, bleeding must be controlled immediately and any tumour "spill" should be avoided. With experience, the laparoscopic technique can be used in treating Wilms' tumour of selected cases. The National Wilms' Tumor Study Group (NWTSG) in the United States recommends that all adult patients with favourable histology should be treated with stage-appropriate combined therapy, as is done for children. Systemic chemotherapy is required. The most effective are: actinomycin $\mathrm{D}(\mathrm{ACT})$, vincristine (VCR), doxorubicin (ADM), cyclophosphamide (CTX), ifosfamide (IFO), etoposide, and carboplatin. However, their toxicity is higher in adults than in children. ${ }^{8}$ Currently, radiation therapy is usually part of treatment only for more advanced Wilms' tumours (stages III, IV, and V) and for some earlier stages tumours with unfavourable histology.

Preoperative tumour rupture can be spontaneous or occur after trauma. Spontaneous rupture of renal tumour is uncommon and the most frequent cause is AML, RCC, and leiomyosarcoma. ${ }^{9,10}$ Spontaneous rupture of a Wilms' tumour is rare. In an analysis of 606 patients with Wilms' tumour, $3 \%$ showed preoperative rupture, including both chronic disruption of the capsule by tumour growth and acute rupture with hemorrhage. ${ }^{11}$ Adult Wilms' tumour as a

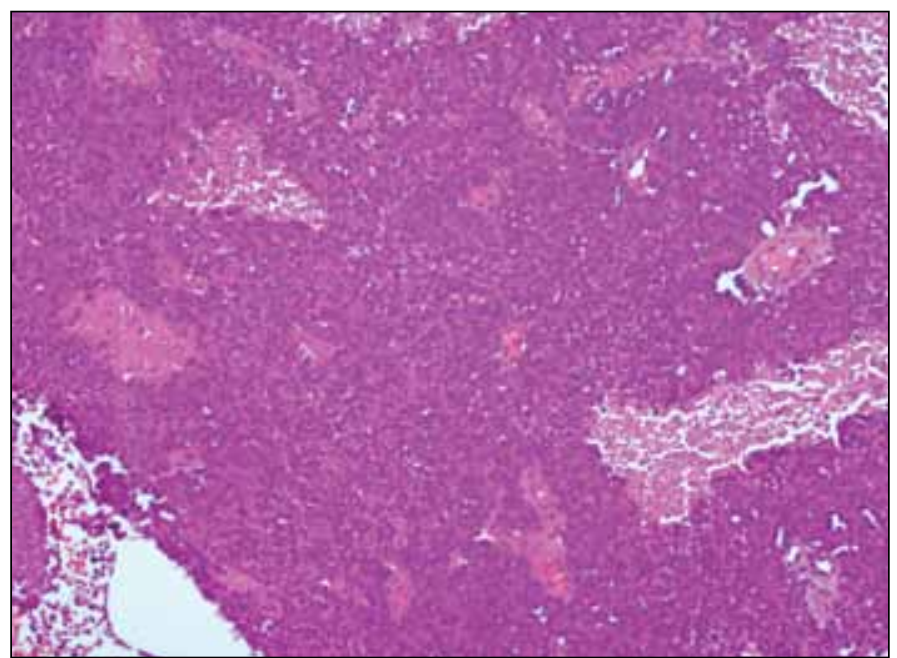

Fig. 3b. Microscopical picture (hematoxylin-eosin, $\times 100$ ) showing blastemal cells and stromal element.

spontaneous rupture is extremely rare. Heyns and Rossouw ${ }^{3}$ presented a 22-year-old man with spontaneous rupture of a Wilms' tumour, who died within 5 months. The authors considered preoperative tumour spillage, unfavourable histology, and the omission of radiotherapy owing to poor patient compliance.

As tumour rupture is a major risk factor for abdominal recurrence, according to current International Society of Pediatric Oncology (SIOP) recommendations, all preoperative tumour ruptures should be classified as stage III and treated with three-drug therapy and whole abdominal radiation. However, Brisse and colleagues ${ }^{12}$ believed patients who have radiologic signs of localized retroperitoneal-only rupture at diagnosis most likely should not be upstaged, and their treatment should be determined according to pathologic stage only. The use of CT scans significantly increased the number of patients who could be classified with tumour rupture, but macroscopic (surgical or pathologic) definition of 'rupture' currently remains the gold standard. Khanna and colleauges ${ }^{13}$ reported CT has moderate specificity $(88 \%$, $61 / 69$ cases), but relatively low sensitivity ( $54 \%$ and $70 \%, 36$ and 47 of 67 cases, respectively) in the detection of preoperative Wilms' tumour rupture. In a study, among those who had ruptures classified as retroperitoneal-only on $\mathrm{CT}$, only 22 of 48 patients were staged as stage III, Consequently, 26 patients who presented radiologic signs of retroperitonealonly rupture at diagnosis were treated postoperatively for stage I disease (17 patients) or stage II disease (9 patients). Therefore, patients with stage I or II Wilms' tumour who have localized retroperitoneal-only rupture at diagnosis may be treated less aggressively than patients with stage III disease, notably without radiotherapy. At stage I, our patient received only 18 weeks of adjuvant chemotherapy with vincristine and actinomycin $D$. 
It is also recommended that clinically stable patients should be treated by neoadjuvant chemotherapy and secondary surgery, which may reduce the risk of tumour rupture during surgery and reduce the probability of local and distant recurrence. However, neoadjuvant chemotherapy is hard to perform in adult patients because of the rarity of Wilms' tumour in adults and its diagnostic difficulty.

The reported prognosis is worse for adults compared with children because the disease is more advanced in adults at the time of diagnosis. The prognosis depends on the primary advancement stage, the histopathology, and time since the first remission, type of therapy, and the recurrence location. The cure rates for adult Wilms' tumour are improving. In 2003, Reinhard and colleagues ${ }^{14}$ reviewed 30 adult patients who were treated with surgery and chemotherapy, as well as 14 out of 30 patients receiving irradiation. They reported a 4 -year median overall survival rate of $83 \%$.

There were some limitations in the diagnosis and treatment of our patient. She had suffered the disease for 2 months before being referred to our hospital, and the first diagnosis of an AML was made without evidence of fat seen on CT. While her condition was considered stable, the patient was at a high risk for further rupture after a long follow-up. Furthermore, a ruptured/bleeding neoplasm was considered an $\mathrm{AML}$, but embolization was not considered. After being at our hospital, a diagnosis of RCC was made, but Wilms' tumour and neoadjuvant chemotherapy were not considered. In addition, the rupture may have been more extensive initially and it was potentially downplayed on evaluation of a single initial axial imaging study. Our patient's disease could have been upstaged to stage III and radiation therapy could have been initiated since local occurrence was hard to confirm.

\section{Conclusion}

Spontaneous rupture of adult Wilms' tumour is extreme rare, which may lead to a bad prognosis with the possibility of tumour spillage. Although the preoperative diagnosis is very difficult, Wilms' tumour should be considered in the differential diagnosis. Radical nephrectomy and postoperative comprehensive therapy are equally important. Systemic chemotherapy or combination with radiotherapy should be given according to pathologic stage. Therapy schemes in adults based on pediatric protocols lead to similar results as observed in children.

Competing interests: The authors all declare no competing financial or personal interests.

This paper has been peer-reviewed.

\section{References}

1. Wein AJ, Kavoussi LR, Novick AC. Campbell-Walsh Urology. 10th ed. Philadelphia: PA, Saunders-Elsevier; 2012

2. Mitry E, Ciccolallo L, Coleman M P, et al. Incidence of and survival from Wilms' tumour in adults in Europe: data from the EUROCARE study. EurJ Cancer 2006;42:2363-8. http://dx.doi.org/10.1016/i. ejca.2006.04.009

3. Heyns CF, Rossouw DJ. Spontaneous rupture of adult Wilms' tumor. Cancer 1989;64:173-7. http:// dx.doi.org/10.1002/1097-0142(19890701)64:1<173::AID-CNCR2820640129>3.0.C0;2-0

4. Kilton L, Matthews MJ, Cohen MH. Adult Wilms tumor: A report of prolonged survival and review of literature. J Urol 1980;124:1-5

5. Dykes EH, Marwaha RK, Dicks-Mireaux C, et al. Risks and benefits of percutaneous biopsy and primary chemotherapy in advanced Wilms' tumour. J Pediatr Surg 1991;26:610-12. http://dx.doi. org/10.1016/0022-3468(91)90719-A

6. Huszno J, Starzyczny-Slota D, Jaworkka M, et al. Adult Wilms' tumor - diagnosis and current therapy. Cent European J Uro, 2013;66:39-44. http://dx.doi.org/10.5173/ceju.2013.01.art12

7. Okasho K, Nishiyama H, Watanabe J, et al. Adult wilms tumor in the renal pelvis: Case report with review of the literature. Urology 2008;72:1185. http://dx.doi.org/10.1016/i.urology.2008.01.036

8. Kumar $A$, Lal B, Singh $M$, et al. Adult Wilms' tumour: Report of a case and review of the literature. Jpn J Surg 1990;20:585-89. http://dx.doi.org/10.1007/BF02471017

9. Grasso $M$, Blanco $S$, Fortuna $F$, et al. Spontaneous rupture of renal leiomyosarcoma in a 45-year-old woman. Arch Esp Urol 2004;57:870-2.

10. Kinjo T, Oida T, Yoneda $S$, et al. Poor outcome due to spontaneous rupture of renal cell carcinoma: A case report. Hinyokika Kiyo 2013;59:517-21.

11. Leape LL, Breslow NE, Bishop HG. The surgical treatment of Wilms' tumor: Results of the national Wilms' tumor study. Ann Surg 1978;187:351-6. http://dx.doi.org/10.1097/00000658-197804000-00001

12. Brisse HJ, Schleiermacher G, Sarnacki S, et al. Preoperative Wilms tumor rupture: A retrospective study of 57 patients. Cancer 2008;113:202-13. http://dx.doi.org/10.1002/cncr.23535

13. Khanna $G$, Naranjo A, Hoffer $F$, et al. Detection of preoperative Wilms tumor rupture with CT: A report from the Children's Oncology Group. Radiology 2013;266:610-7. http://dx.doi.org/10.1148/ radiol. 12120670

14. Reinhard H, Aliani S, Ruebe C, et al. Wilms' tumor in adults: Results of the Society of Pediatric Oncology (SIOP) 93-01/Society for Pediatric Oncology and Hematology (GPOH) Study. J Clin Oncol, 2004;22:4500-6. http://dx.doi.org/10.1200/JC0.2004.12.099

Correspondence: Dr. Jian-lin Huang, Department of Urology, Sichuan Academy of Medical Sciences

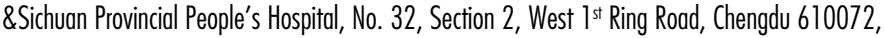
Sichuan, People's Republic of China, hil319@126.com 